\title{
Tinbergen and Tipping points: Could some thresholds be policy-induced?
}

\author{
Eli P. Fenichel ${ }^{1}$ and Richard D. Horan ${ }^{2}$ \\ ${ }^{1}$ Yale School of Forestry \& Environmental Studies, eli.fenichel@yale.edu (corresponding author) \\ ${ }^{2}$ Michigan State University, Department of Agricultural, Food, and Resource Economics, horan@msu.edu \\ Authorship is equally shared and alphabetical
}

Acknowledgements: EPF is supported by National Foundation grant no DEB-1213693. EPF and RDH were supported by NSF grant 1414374. RDH was also supported by USDA National Institute of Food and Agriculture grant 2011-67023-30872 and AgBioResearch of Michigan State University. T.N. Fenichel (age 3.5 months at initial time of writing) has provided E.P. Fenichel with new insights into the meaning of tipping points, for which he is grateful.

\begin{abstract}
Thresholds and tipping points that are characteristic of dynamic multi-stability are increasingly prominent touchstones of the science-policy interface. Prior economic work has shown thresholds arising in convex-concave ecological models that exclude humans may disappear in models of coupled economic-ecological systems when economic agents are included as part of the system. Less wellunderstood is how this result may depend on the ability of humans to manage complex systems. We examine the role of institutions that condition the available management choices. We find institutional restrictions do not generate non-convexities when the ecological system is well-behaved, but that these restrictions may generate non-convexities when the ecological system is convex-concave. These results are tied to the concept of path controllability, which is when managers have sufficient controls to guide the system across the state space. Path controllability is the dynamic version of the Tinbergen Rule, and generally requires separate controls to manage each possible ecological externality. We link the concepts of ecosystem externalities, thresholds, and path controllability to provide new economic insights into problems with the potential for multi-stability, and we illustrate the importance of institutions for managing convex-concave systems.
\end{abstract}

Keywords: Optimal control, Skiba point, sufficiency conditions, path controllability, polycentric governance, decouplability, socio-ecological system (SES), ecological-economic model, institutions

JEL codes: C61, C63, Q20, Q57 


\section{Tinbergen and Tipping points: Could some thresholds be policy-induced?}

\section{Introduction}

Micro processes combine to produce macro phenomena that are difficult to anticipate (Chen, 2002), and complexity is further increased when ecological and economic systems are considered jointly (Levin et al., 2013). The complex nature of systems leads to concerns in economics (Skiba, 1978; Starrett, 1972) and ecology (Beisner et al., 2003) about non-unique long-run outcomes, often expressed as multiple equilibria that are separated in the state space by economic thresholds or tipping points. Economic thresholds are policy relevant because they suggest the long-run nature of the system, and the net benefits the system provides, hinge on the initial conditions at the time when a new management program is introduced. Therefore, the timing of when policy changes occur (or where policy change occurs in state space) can critically influence outcomes.

Ecological multi-stability has attracted a great deal of attention in the ecological literature (Biggs et al., 2009; Groffman et al., 2006; Lenton et al., 2008; Mumby et al., 2007; Scheffer and Carpenter, 2003), and the underlying non-convexities are of increasing interest in environmental and resource economics (Dasgupta and Maler, 2003 and other papers in this issue). Economists have shown that economic multi-stability arises in natural resource problems due to non-convex state equations or economic relations (Brock and Starrett, 2003; Davidson and Harris, 1981; Horan and Bulte, 2004; Rondeau, 2001; Tahvonen and Salo, 1996; Wirl, 2004). However, less work has addressed how economic multi-stability and thresholds emerge through ecological interactions (Tschirhart, 2012) or the role of institutional arrangements in managing these interactions (Horan et al., 2011). This is despite Wirl and Feichtinger's (2005) plea to carefully motivate non-convexity.

Efficient management of complex coupled systems requires managing a potentially large number of ecological interactions, as ecosystems are not inherently efficient providers of services. Indeed, ecological agents can easily dissipate "ecological rents" without human interventions to 
preserve these rents (Horan et al., 2011; Melstrom and Horan, 2013), thereby generating a form of ecosystem externality — when one ecological variable indirectly and inefficiently impacts other ecological variables through ecological interactions (Crocker and Tschirhart, 1992). Efficiency requires these ecosystem externalities be targeted by management, but there may be too many complex interactions to target all ecosystem externalities. ${ }^{1}$

We investigate the implications of imposing restrictions on the control set for ecosystem management, with a particular focus on the implications for multi-stability. In particular, we find that institutional limitations on the set of available policy variables may reduce the path controllability (Aoki and Canzoneri, 1979; Nyberg and Viotti, 1978) of non-convex ecological systems, where path controllability in this context refers to managers' ability to guide ecological systems across the state space. This result extends Aoki and Canzoneri's and Nyberg and Viotti's original macroeconomic applications, with savings and money supply as state variables, and wages and interest rates as the control variables. The core problem these authors investigate is whether only controlling interest rates is sufficient to optimally manage an economy; they find it is not, except for some special cases. In our extension of this work, we show that institutional limitations on the set of available control variables does not simply lock in second-best decision making (Lipsey and Lancaster, 1956), but possibly generates economic multi-stability. Alternatively, we find multi-stability may vanish when managers can benefit from more flexible institutions that expand the control set to improve the path controllability of the system.

Our results are related to the Tinbergen rule (Tinbergen, 1956) which states that managers must adopt separate policy instruments to manage each externality. Indeed, the concept of path

\footnotetext{
${ }^{1}$ The notion of targeting all the interactions of the various ecological stocks is somewhat akin to targeting the multiple tasks of interacting agents (Holmstrom and Milgrom 1991). In particular, Holmstrom and Milgrom find that interactions among tasks generate non-convexities in non-symmetric solutions, which must be addressed to create the correct incentives. They also indicate that the many tasks need to be targeted in their totality to encourage an efficient allocation of efforts. Our analysis goes beyond this approach to consider optimal management when regulators are restricted in their targeting abilities.
} 
controllability (and the related concept of decouplability; Aoki and Canzoneri 1979) generalizes the Tinbergen rule to dynamic economic systems (Aoki and Canzoneri, 1979; Nyberg and Viotti, 1978). We show the Tinbergen rule extends to ecosystem externalities. If ecosystem externalities are allowed to persist in the system, then by definition the system is second-best. However, given the breadth of potential ecological interactions it is highly unlikely that all ecosystem externalities can be eliminated completely. The critical question is when does targeting an externality change the coupled dynamics in a non-marginal way to qualitatively alter the long-run economic and ecological outcomes? The answer to this question cannot be answered in a general way, but the answer appears to sit at the intersection of concerns over convexity and path controllability. We extend prior work by more formally considering how relaxing certain assumptions, long used to avoid 'ill behaved' models with multiple equilibria, affects management opportunities and may lead to systems that are at best only partially path controllable. This analysis helps to clarify when multi-stability is a fundamental component of a coupled system versus when multi-stability may emerge as the result of modeling simplifications or institutional constraints. We focus on the management of ecological systems, but our results are relevant for broad range of dynamic public economic problems.

\section{System description}

We consider the canonical natural resource economics management problem, but explicitly expand the problem to involve two interacting state variables expressed as the state vector $s=\left[s^{x} s^{y}\right]^{\mathrm{T}}$, thereby illustrating the simplest possible managed ecosystem. Ecosystem dynamics are given by the system

$$
\dot{\boldsymbol{s}}(t) \equiv\left[\begin{array}{c}
\dot{s}^{x}(t) \\
\dot{s}^{y}(t)
\end{array}\right]=\boldsymbol{G}(\boldsymbol{s}(t))-\boldsymbol{c}(t) \equiv\left[\begin{array}{l}
G^{x}\left(s^{x}(t), s^{y}(t)\right) \\
G^{y}\left(s^{x}(t), s^{y}(t)\right)
\end{array}\right]-\left[\begin{array}{l}
c^{x}(t) \\
c^{y}(t)
\end{array}\right]
$$

where $G^{x}$ and $G^{y}$ represent net growth of the states prior to accounting for management by the harvest 
controls $c^{x} \geq 0$ and $c^{y} \geq 0 .{ }^{2}$ We assume there are interactions among the states in $\mathbf{G}$ such that $\partial G^{i} / \partial s^{j} \neq 0$ for at least one $i \in\{x, y\}$ with $i \neq j$.

The management problem associated with (1) is defined as

$$
\begin{aligned}
& V=\max _{c^{i} \in \Omega} \int_{0}^{\infty} W\left(s^{x}, s^{y}, c^{x}, c^{y}\right) e^{-r t} d t \\
& \text { s.t. } \quad(1) ; \quad \mathbf{s}(0)=\mathbf{s}_{0} ; \mathbf{c}(t) \geq \mathbf{0}
\end{aligned}
$$

where $W$ represents the net economic benefits in each period, $r$ is the discount rate, and $\Omega$ is the set of feasible controls that the social planner has the ability to adjust in response to the state of the system. We assume $W$ and $\mathbf{G}$ are specified such that utilizing both controls, i.e., $\Omega=\left\{c^{x}, c^{y}\right\}$, rather than a corner solution involving one or no controls, would be efficient or first-best. ${ }^{3}$

We consider two cases associated with $\Omega$ : (i) a first-best scenario with a complete control set, $\Omega$ $=\left\{c^{x}, c^{y}\right\}$, and (ii) a second-best scenario with an incomplete control set, e.g., $\Omega=\left\{c^{x}\right\}$, such that $c^{y}=\bar{c}^{y}$ is fixed. The second-best case represents a system where only a subset of state variables are being actively managed in response to changes in $\mathbf{s}$ (hence, we refer to $\Omega$ as the set of active controls). This can occur when institutions are restricted or otherwise incapable of providing the optimal management of $c^{y}$. This setup reflects the more general result that ecosystems have many interacting components that generate ecosystem externalities, and it is hardly reasonable to think that managers

\footnotetext{
${ }^{2}$ More generally, we could write $\dot{\boldsymbol{s}}(t)=\boldsymbol{J}(\boldsymbol{s}(t), \boldsymbol{c}(t))$, where $\boldsymbol{J}$ now represents the net effects of both ecological and anthropogenic interactions, including non-harvest activities such as supplemental feeding of wildlife, alternative forms of habitat provision, or land use affecting population growth. Some of these human choices could interact nonlinearly with state variables. The most general case would involve thinking of $\mathbf{c}$ not as the actual human choices but rather as a vector of regulatory tools (e.g., taxes or tradeable quotas) that influence (or compel) the particular decisions made by individuals within an economy (e.g, see Fenichel and Abbott (2014a) for an example of this approach). We focus on the linear form in (1), $\boldsymbol{J}(\boldsymbol{s}(t), \boldsymbol{c}(t))=G(\boldsymbol{s}(t))-\boldsymbol{c}(t)$, to simplify the exposition, but we note in a series of footnotes that the results generally apply to the nonlinear case.

${ }^{3}$ For instance, we rule out the following two cases (there may be others) where a corner solution with $c^{i}=0 \forall t$ would arise for $i \in\{x, y\}$ with $i \neq j: \partial W / \partial c^{y}<0$ is sufficiently negative $\forall c^{y}$, or else $\partial W / \partial s^{i}>0, \partial W / \partial s^{i}>0$, $\partial G^{j} / \partial s^{i}>0 \forall s^{i}$ and $\partial W / \partial c^{i}$ is sufficiently small for small $c^{i}$.
} 
will control all of these interactions in targeted ways. ${ }^{4}$ This means that the optimized path of $\boldsymbol{s}(t)$ under an incomplete control set generally deviates from the first-best path so as to yield a smaller $V$ than in the first-best case.

The current value Hamiltonian for the first-best and second-best scenarios is

$$
H(\boldsymbol{s}, \boldsymbol{c}, \lambda)=W(\boldsymbol{s}, \boldsymbol{c})+\lambda^{T}[\boldsymbol{G}(\boldsymbol{s})-\boldsymbol{c}]
$$

where $\lambda=\left[\begin{array}{ll}\lambda^{x} & \lambda^{y}\end{array}\right]^{\mathrm{T}}, \lambda^{i}$ is the co-state associated with state $s^{i}$, and where the restriction $c^{y}=\bar{c}^{y}$ is imposed exogenously in the second-best case. ${ }^{5}$

\subsection{Conventional assumptions}

We follow the literature and make several conventional assumptions about problem (2). First, we assume $W$ is nonlinear in the controls, although our core results generalize to problems linear in the controls (Horan et al., 2011). ${ }^{6}$ Second, we assume additive separability of the controls in W. Additive separability is not required, but it is a common assumption in natural resource problems and greatly

\footnotetext{
${ }^{4}$ For instance, managers often focus on managing species that provide direct values, such as through hunting, fishing, wildlife viewing, or existence values. Fenichel et al. (2010) examine such a case where managers have some control over valued predators (salmon in Lake Michigan) but not unmarketable prey (alewife). Another example is that managers may be able to target habitat investments to protect an endangered species (piping plovers) from a predator (merlins, a small falcon), but are unable to harvest the predator due to the predator also being a threatened species (Melstrom and Horan 2014). Our qualitative results also extend to a slight variation of this second-best framework, which involves expanding the use of the managed control $\left(c^{x}\right)$ to affect multiple states. For instance, a second-best outcome arises when a single control, $c^{x}$, is applied non-selectively to heterogeneous ecosystem components. This could include harvesting individuals of a fish or wildlife population without regard to important attributes that influence dynamics, such as sex, age, or disease status (Fenichel and Horan 2007a,b) when in fact it would be more efficient to target these components selectively. More generally, habitat may be provided in a broad sense, such as through reserves or other set-asides, to impact multiple species. But little-to-no effort may be devoted to managing the many complex interactions occurring on these lands, such as predation or the availability of prey when these other species provide only indirect values through ecological interactions with the valued species.

${ }^{5}$ We focus on the Hamiltonian rather than on a Lagrangian that takes the non-negativity constraints into consideration, because we assume the non-negativity constraints do not bind, and hence the associated multipliers vanish.

${ }^{6}$ We focus on nonlinear control problems because this allows us to work with sufficiency conditions, and we make extensive use of sufficiency conditions below.
} 
simplifies the analysis by requiring $W_{c x, c y}=0{ }^{7}$

A final conventional assumption about problem (2) is that the Hamiltonian is strictly concave, such that this problem is strictly convex, in the first-best setting. This assumption ensures the first-best solution at each initial state $\mathbf{s}_{0}$ is unique (Caputo 2005), implying no indifference (Skiba, 1978) manifolds exist in the state space. This means there are no locally optimal (or history-dependent) solutions; the first-best outcome is characterized by a single basin of attraction for a unique, globally optimal long-run outcome. $^{8}$

We assume strict convexity of the problem for the first-best case, not to suggest that all firstbest problems are strictly convex or that strict convexity is necessary for a global long-run optimum (indeed, a global optimum is possible even in problems that are not strictly convex; e.g., Wagener, 2003). Rather, this assumption allows us to clearly illustrate the potential convexity implications of restricting the control set in the second-best case: if the first-best is strictly concave and the second-best is not, then any non-convexities and multiple equilibria arising in the second-best case must be due to the altered assumptions of the second-best case. Specifically, we will show how a restricted control set, leading to second-best management, can create non-convexities in some settings to generate multiple equilibria. ${ }^{9}$ This is not the only way in which multiple equilibria can arise (Wirl and Feichtinger, 2005), but we show that it is a potentially important source of multiple equilibria in coupled ecologicaleconomic systems. This is especially true when the ecological growth function $\mathbf{G}(\mathbf{s})$ is convex-concave, which as we show below and in Table 1 is common in models of ecological interactions.

Two different notions of sufficiency can be used to verify strict convexity, ensuring uniqueness

\footnotetext{
${ }^{7}$ We denote $F_{c x}=\partial F / \partial c^{x}$ for each function $F$. Higher order derivatives are written similarly.

${ }^{8}$ It may be possible to have multiple equilibria satisfying the first order necessary conditions, but one will be a global optimum. Skiba points can exist in a single dimension. These thresholds are referred to as Skiba manifolds when they exist in higher dimensions (Caulkins et al., 2009; Horan et al., 2011). Skiba manifolds are also referred to as Dechert,Mishimura, Sethi, and Skiba (DNSS) manifolds, and a general theory does not yet exist for systems greater than one dimension (Caulkins et al., 2009).

${ }^{9}$ Our results suggest that restrictions on the control set may exacerbate non-convexities in situations where the first-best problem also exhibits non-convexities.
} 
(Leonard and Long 1998; Caputo 2005). The most general notion of sufficiency, but also the most difficult to verify, is Arrow sufficiency. Arrow sufficiency requires the optimized Hamiltonian, $H^{*}(\mathbf{s}(t), \lambda(t)) \equiv \max _{\mathbf{c}} H(\mathbf{s}, \mathbf{c}, \lambda) \forall t$ to be strictly concave in $\mathbf{s}$ when $\lambda(t)=\lambda^{*}\left(t ; \mathbf{s}_{0}\right)$ is the first-best costate given the initial state $\mathbf{s}_{0}$. This condition ensures that a unique, long-run maximizing equilibrium can emerge regardless of any non-convexity in the ecological dynamics (Horan et al., 2011; Tahvonen and Salo, 1996). The more common and easier-to-verify, but less general, type of sufficiency is Mangasarian sufficiency. This requires $H(s, c, \lambda)$ to be strictly concave in the states and controls when $\lambda(t)=\lambda^{*}\left(t ; s_{0}\right)$ (e.g., Caputo 2005). A common practice in dynamic economics is to make particular concavity assumptions about the relations $W$ and $\mathbf{G}$ in order to satisfy the Mangasarian sufficiency conditions. The specific assumptions, as they apply to problem (2), are described in the following section 2.2.

\subsection{Generalizing assumptions}

We make several generalizing assumptions that relax assumptions often implicitly or explicitly made in traditional multi-state problems. ${ }^{10}$ First, we assume there are interactions among state variables. Allowing for state interactions contrasts with assumptions traditionally made in multi-state economic problems, although interactions are increasingly modeled in bioeconomic applications (e.g., Fenichel et al., 2010; Horan et al., 2011; Wirl, 1992).

Second, we do not restrict the co-states to be positive, so that each state variable $i$ can be either an asset, $\lambda^{i}>0$, or a liability, $\lambda^{i}<0$, at any time $t$. Note that $W_{c i}>0$ in the case of valuable harvests $\left(\lambda^{i}\right.$ $>0)$, whereas $W_{c i}<0$ when $s^{i}$ is harvested as a nuisance species $\left(\lambda^{i}<0\right)$.

Third, as indicated above in our discussion of the second-best scenario, we relax the standard

\footnotetext{
${ }^{10} \mathrm{An}$ additional generalizing assumption for the more general case where $\boldsymbol{J}$ is nonlinear in the controls is that each control may affect multiple states (Fenichel and Horan, 2007b; Wirl, 1992). This assumption would not affect the qualitative results described here.
} 
assumption that there must be one actively managed control for each state, as managers generally do not target all of the relevant ecological interactions within a system. This means the set of feasible active controls, $\Omega$, is restricted such that there are fewer active controls than states. Specifically, we assume exogenous restrictions have been placed on the control $c^{y}$ such that the manager must choose $c^{x}$ accounting for the predetermined value of $c^{y}$ (Fenichel and Abbott, 2014a; Lipsey and Lancaster, 1956). ${ }^{11}$

Finally, we relax some assumptions that are typically made to ensure the management problem is convex. ${ }^{12}$ Specifically, in the context of problem (2), the traditional assumptions are that $W$ and $G^{x}$ are strictly concave in the states and controls (given that $\lambda^{x}>0$ ), and $G^{y}$ is strictly concave (convex) in the states and controls with $\lambda^{y}>0\left(\lambda^{y}<0\right)$. We refer to these assumptions as the Mangasarian assumptions because they ensure the problem satisfies the Mangasarian sufficiency conditions for strict convexity and uniqueness of the optimizing equilibrium. We assume the Mangasarian assumptions hold in section 3 to illustrate the properties of the first-best and second-best solutions in such settings, including the result that non-convexities do not arise when $\Omega$ is restricted.

The Mangasarian assumptions are likely to be too strong in the case of many realistic ecosystem interactions represented by $\mathbf{G}(\mathbf{s})$ (and Skiba (1978) argues this is also true for many real world economies). Table 1 illustrates several standard ecological models of two-species interactions, each of which yields Hessian matrices that are not negative (or positive) definite. Specifically, the Lokta-Volterra model is the standard competition model used in ecology, whereas predator-prey relations are typically modeled using the Holling Type I, II and III predation responses (Gotelli, 2008), with Type I responses also being the most commonly used specification for modeling infectious disease problems (with $x$ and $y$ indexing the susceptible and infected populations, respectively). Most ecological models, and

\footnotetext{
${ }^{11}$ Alternatively, the value of $c^{y}$ could be constrained at the boundary of the control set, e.g., $c^{y}=0$ if $c^{y} \in \mathbb{R}^{+}$, but this would be observationally equivalent. One important case is where the controls are chosen by uncoordinated independent organizations.

${ }^{12}$ Note that a (strictly) convex problem implies a (strictly) concave (or strictly convex) Hamiltonian, whereas the problem is non-convex iff the Hamiltonian is convex-concave.
} 
specifically ecological models used in economics, are built up from these core relationships (Fenichel et al., 2015; Wilen, 1985). The canonical versions of these models require two state variables, though it is common to build higher dimensional systems using these or similar relationships (e.g., Brock and Xepapadeas, 2002). If more complex ecosystems are built up from these interactions, then their Hessian matrices will also not be negative (or positive) definite.

Obviously, the Mangasarian assumptions cannot hold in bioeconomic analyses involving convexconcave forms of $\mathbf{G}(\mathbf{s})$. In such instances, rather than imposing countervailing assumptions to ensure the Arrow sufficiency conditions are satisfied, prior bioeconomic work has largely examined when the nonconvexities associated with $\mathbf{G}$ generate multiple equilibria (e.g., Maler et al. 2003; Horan et al. 2011). We take a different approach due to our interest in the effects of restricting the control set $\Omega$. Specifically, in section 4 we consider convex-concave forms of $\mathbf{G}(\mathbf{s})$ for cases where the Arrow sufficiency conditions are satisfied for the first-best problem, thereby ensuring the first-best problem is still strictly convex. Importantly, such cases exist when the control set is complete even when the system dynamics are not strictly concave. However in this setting, we find that the Arrow sufficiency conditions may not be satisfied in the second-best problem, so that restrictions on $\Omega$ potentially yield non-convexities and multiple equilibria. This highlights the role of the choice set and institutional restrictions in generating non-convexities.

\section{First-best and second-best outcomes under the Mangasarian assumptions}

Suppose the Mangasarian assumptions are satisfied. Then, as described above, the Hamiltonian with the complete control set is strictly concave and the optimal solution for a particular $\mathbf{s}_{0}$ is unique with a single basin of attraction for a unique long-run outcome, regardless of $\mathbf{s}_{0}$. For the following analysis it will be helpful to denote the first-best control by $\mathbf{c}^{*}\left(t ; \mathbf{s}_{0}\right)$, which can be written in feedback form as $\mathbf{c}^{*}(\mathbf{s}(t))$. The associated welfare level is $V^{*}\left(s_{0}\right)$. 


\subsection{Path controllability and decouplability in the first-best outcome}

Controllability is an important concept that has been under-investigated in resource economics (Fenichel et al., 2015). There are two notions of controllability. The first, typically presented simply as controllability, (e.g., Bryson and Ho, 1975; Caputo, 2005), is more precisely called point controllability. Point controllability is the ability to traverse the state space from any initial condition to any particular state in finite time. For instance, suppose managers faced an exogenously-defined goal of $s^{x}$ being doubled at $t=10$. The system would be point controllable if this goal was feasible, and it would not be point controllable otherwise. As the point controllability property only ensures the system's trajectory can pass through a desired point at some finite time, this property has limited relevance for infinite horizon problems and economic planning more generally. A particular concern for infinite horizon problems is that a point controllable trajectory does not guarantee the target point can be maintained (e.g., as a steady state) once it is reached, nor does it guarantee that the system is otherwise easily managed after the target date (Nyberg and Viotti, 1978). Nyberg and Viotti (1978) liken the notion of point controllability to controlling a missile's trajectory: a missile can be guided to explode at a target, but there is no regard for where the missile's trajectory would have led after the explosion. Absent sufficient controllability of the system after attaining the target point, binding restrictions may limit the ability to control what happens next, producing inefficiencies.

The second notion of controllability is perfect or path controllability, which stipulates the controls enable managers to follow any given path over the entire infinite planning horizon (Aoki and Canzoneri 1979). Managers of a path controllable system are able to determine an optimal trajectory and follow it without restriction. In contrast, a system that does not exhibit path controllability may involve efficiency-reducing restrictions on the trajectories that managers might pursue to control the system. Path controllability implies point controllability, but the converse is not true (Aoki and 
Canzoneri, 1979). Path controllability is a stronger requirement than point controllability, but it is clearly also the relevant property for managing an infinite horizon problem.

A mathematical definition of path controllability begins by following Aoki and Canzoneri (1979) to define a state space representation. This consists of the vector-based state equation (1) and a "target equation", which in our case is a two-dimensional target vector

(4) $\mathbf{q}(t)=\mathbf{s}(t)$

Our target vector is a special case of Aoki and Canzoneri's (which is defined as a linear function of states and controls), as our interest is simply in the ability of a control to move the system around the state space. Specifically, the system (1) and (4) is said to be path controllable if there exists a control function, $\mathbf{c}(t)$, that can guide $\mathbf{q}(t)$ from any initial point along any differentiable time path $\mathbf{P}(t)$ in two dimensional space, over any arbitrary time horizon. A necessary condition for path controllability is that the number of controls is not less than the number of states (Aoki, 1975), which is a dynamic form of the Tinbergen (1956) rule (Aoki, 1975; Aoki and Canzoneri, 1979; Nyberg and Viotti, 1978). This condition is satisfied in the first-best case.

Aoki and Canzoneri (1979) present sufficient conditions for path controllability, with their condition B (p.607) being applicable for our target vector (4). Specifically, their condition B states that it is possible to solve the state equation for the control rule that guides the state vector along any given target path $\mathbf{P}(t)$. The linear nature of (1), with the implicit coefficient of $c(t)$ being $-\boldsymbol{I}$ (i.e., the negative of the identity matrix), ensures we can solve for this control rule. Specifically, the control rule is obtained by setting $\boldsymbol{s}=\boldsymbol{P}$ and $\dot{\boldsymbol{s}}=\dot{\boldsymbol{P}}$ and then solving equation (1) for $\boldsymbol{c}(t)=\dot{\boldsymbol{P}}(t)-\boldsymbol{G}(\boldsymbol{P}(t)) .{ }^{13}$ This control rule

\footnotetext{
${ }^{13}$ For a more general form of our model in which $\boldsymbol{J}$ is nonlinear in the controls, Aoki and Canzoneri (1979) indicate their sufficiency condition B implies local path controllability for linearized versions of $\boldsymbol{J}$ in the neighborhood of $\mathbf{P}(t)$. For instance, consider linearizing the nonlinear version of $\boldsymbol{J}$ around the point $\left(\underline{s}^{\sharp}(t), \underline{\mathbf{c}^{\sharp}}(t)\right)=(\boldsymbol{P}(t), 0)$ such that . $\boldsymbol{J} \approx \boldsymbol{J}(\boldsymbol{P}(t), \mathbf{0})+\nabla_{S} \boldsymbol{J}(\boldsymbol{P}(t), \mathbf{0})(\boldsymbol{s}(t)-\boldsymbol{P}(t))+\boldsymbol{\nabla}_{\boldsymbol{c}} \boldsymbol{J}(\boldsymbol{P}(t), \mathbf{0}) \boldsymbol{c}(t)$. Then the applicable sufficiency condition is $\left|\boldsymbol{\nabla}_{\boldsymbol{c}} \boldsymbol{J}(\boldsymbol{P}(t), \mathbf{0})\right| \neq 0$, such that the control rule $\boldsymbol{c}(t)=\left(\boldsymbol{\nabla}_{c} \boldsymbol{J}(\boldsymbol{P}(t), \mathbf{0})\right)^{-1}\left[\dot{\boldsymbol{P}}(t)-\boldsymbol{J}(\boldsymbol{P}(t), \mathbf{0})-\boldsymbol{\nabla}_{\boldsymbol{S}} \boldsymbol{J}(\boldsymbol{P}(t), \mathbf{0})(\boldsymbol{s}(t)-\right.$ $\boldsymbol{P}(t))]$ is satisfied for all possible $\boldsymbol{P}(t)$. It is not possible to determine whether the sufficiency condition $\left|\nabla_{\boldsymbol{c}} \boldsymbol{J}(\boldsymbol{P}(t), \mathbf{0})\right| \neq 0$ is satisfied for all feasible $\boldsymbol{P}(t)$ without imposing further structure on our model. However,
} 
is unlikely to hold for all $\boldsymbol{P}(t)$ since $\mathbf{c}(t)$ is constrained to be non-negative. However, problem (2) is considered path controllable for the subset of paths $\boldsymbol{P}(t)$ that do not involve negative values of $c(t)$. We refer to this feature as feasible path controllability (FPC). FPC is desirable because it means no additional constraints on the set of possible trajectories $\boldsymbol{P}(t)$, other than the non-negativity constraints already identified in problem (2), are implied by the set of available controls $\Omega$ used to manage the dynamical system (1). In contrast, the absence of the FPC property would imply constraints on how the system can be controlled, with these constraints being costly when they are binding.

An important, related concept that helps explain why a control for each state is important is decouplability (Aoki and Canzoneri, 1979). Decouplability means that the system (1) and (4) can be transformed into a set of two independent equations that can each be managed by a single control. ${ }^{14}$ The implication is that each state can be managed without spillover effects affecting the other state. Otherwise, efforts to manage one state will have unintended impacts on the other, which generally increases the cost of controlling the system (and can lead to non-convexities). Decouplability therefore is generally an efficiency-enhancing property. The feature of independent control is also particularly important if management authority lies with different organizations, such as when federal and state governments split authority for species management in a particular ecosystem (e.g., if one species is on the endangered species list). The absence of decouplability means coordination failure could arise in such instances, whereas having separate agencies would not jeopardize the first-best outcome if the

problem (2) is trivially path controllable from all initial states for the first-best path $\mathbf{P}^{*}\left(t ; \mathbf{s}_{0}\right) \equiv \mathbf{s}^{*}\left(t ; \mathbf{s}_{0}\right)$ when $\Omega=$ $\left\{c^{x}, c^{y}\right\}$. This is because path $\mathbf{P}^{*}\left(t ; \mathbf{s}_{0}\right) \forall \mathbf{s}_{0}$ was endogenously determined as the unique optimum (as guaranteed by the Mangasarian conditions) for problem (2), which implies that $\boldsymbol{J}_{c}^{*}\left(\boldsymbol{P}^{*}\left(t ; \boldsymbol{s}_{0}\right), 0\right)$ is non-singular.

${ }^{14}$ This feature is somewhat trivially satisfied in the current model where a single control manages each state. But the property would hold in a first-best outcome even in the more general case where $\boldsymbol{G}$ is a nonlinear function of the controls and where each control can affect multiple states. In this case, decouplabilty ensures the nonlinear system (1) and (4) can be transformed into two independent equations, where each is independently controlled by a new control that is constructed as a linear transformation of the original controls. It is as if there are two independent controls managing each state along the optimal path - even though each control in the untransformed model may affect multiple states (Aoki and Canzoneri, 1979). 
system were decouplable. ${ }^{15}$ Aoki and Canzoneri (1979) indicate that the sufficient conditions (but not necessary conditions) for decouplabiilty are the same as those for path controllability, at least for a linearized version of the system. Therefore, since the problem satisfies the FPC property it is also decouplable. $^{16}$

\subsection{The second-best case with Mangasarian sufficiency}

Now consider the second-best scenario with an incomplete control set, $\Omega=\left\{c^{x}\right\}$ and $c^{y}=\bar{c}^{y}$. ${ }^{17}$ This restriction does not alter the concavity or convexity of $W, G^{x}$, and $G^{y}$ with respect to the other state and control variables. Accordingly, the second-best form of problem (2) remains strictly concave, and the second-best solution, which we denote for the states and control in vector form as $\mathbf{s}^{* *}\left(t ; \mathbf{s}_{0}\right)$ and $c^{x^{* *}}\left(t ; \mathbf{s}_{0}\right)$ (or in feedback form as $\mathbf{c}^{* *}(\mathbf{s}(t))$ ), represents a unique, global optimum yielding welfare of $V^{* *}\left(s_{0}\right)$. In other words, restrictions on control set do not lead to multiple, local long-run optima that depend on history, because the problem remains strictly convex in the Mangasarian sense. ${ }^{18}$ However, restrictions on the control set do influence short-run and long-run outcomes in ways that likely reduce economic welfare, $V^{* *}\left(s_{0}\right)<V^{*}\left(s_{0}\right)$, since welfare must be non-increasing in restrictions on $\Omega$.

Further insight into the nature of the second-best outcome arises from noting that the restriction $c^{y}=\bar{c}^{y}$ means the number of active controls is less than the number of states, and so the system is not path controllable along all possible trajectories $\mathbf{P}(t)$. In particular, the second-best system is not path controllable along the first-best path except for the trivial case where $c^{y}$ is optimally

\footnotetext{
${ }^{15}$ Indeed, this is the macro-economic policy context of Aoki and Canzoneri's original paper.

${ }^{16}$ Problems involving the more general nonlinear $\boldsymbol{G}$ are also decouplable along the first-best trajectory since uniqueness of the first-best outcome implies $\boldsymbol{G}_{\boldsymbol{c}}^{*}\left(\boldsymbol{P}^{*}\left(t ; \boldsymbol{s}_{0}\right), 0\right)$ is non-singular, thereby satisfying the sufficiency condition for decouplability along the first-best path.

${ }^{17}$ Throughout we assume that $c^{y^{*}} \neq \bar{c}^{y}$ for at least some interval of time, such that the restriction on the control set is nontrivial.

${ }^{18}$ It is worth emphasizing that we have assumed the inability of resource managers to control $c^{y}$ does not mean other individuals gain control over this variable. If $c^{y}$ is a choice made by individuals who are not regulated in the second-best case, and if this choice is made as a state-dependent behavior (i.e., $c^{y}(t)=c^{y}(s(t))$ in the second-best case), then non-convexities could arise (Dasgupta and Maler 1993).
} 
constrained at $\bar{c}^{y}$ along the entire first-best path. In all but this unlikely case, the dynamic Tinbergen rule is not operationalized. Imperfect path controllability effectively eliminates a large number of potential trajectories in the state space, including the first-best trajectory and the long-run outcome associated with this trajectory (assuming $c^{y} \neq \bar{c}^{y}$ at the first-best steady state). This means any solution to the second-best problem must follow an alternative path. The intuition is that restrictions on $\Omega$ shrink the set of controllable paths within the state space, thereby imposing efficiency-reducing restrictions on the second-best problem that do not appear explicitly in problem (2). Indeed, the second-best solution amounts to choosing the best path from the subset of controllable paths.

Although restrictions on $\Omega$ shrink the set of potential solutions, these restrictions do not generate multiple, local long-run optima that are history dependent. The second-best path is unique but different than the first-best path. Differences in the first-best and second-best feedback control functions, $\mathbf{c}^{*}(\mathbf{s}(t))$ and $\mathbf{c}^{* *}(\mathbf{s}(t))$, mean the topology of the state space, as characterized by equation (1) after substituting in the appropriate control function for $c(t)$, will differ in the two cases. Topologically, the restrictions on $\Omega$ essentially result in a change in the curvature of the optimal topological landscape as well as the steady state within a unique basin of attraction, although without introducing a tipping point into the system.

\section{Relaxing the Mangasarian assumptions}

\subsection{First-best under Arrow assumptions}

We now relax the Mangasarian assumptions, which generally cannot be satisfied for realistic models of complex systems such as ecosystems (Table 1). Our goal is to show that restrictions on $\Omega$ can yield nonconvexities and potentially generate indifference thresholds (Skiba manifolds) in this setting, even if Arrow sufficiency conditions are satisfied when $\Omega$ is unrestricted. ${ }^{19}$ Given our focus on non-convexities

\footnotetext{
${ }^{19}$ We do not claim this is the only way to get thresholds, but we believe it is an important case.
} 
associated with $\mathbf{G}$, suppose $W$ remains strictly concave in the controls and states, but that one or more of the relations $G^{x}$ and $G^{y}$ is not strictly concave in the states, as is the case for the core ecological population dynamics models shown in Table 1. Further, we make the common, simplifying assumption that there are no interactions between $s^{i}$ and $c^{j}$, for $i, j \in\{x, y\}$ and $i \neq j$, so that $H_{s i, c j}=0$. We assume the Arrow sufficiency conditions hold in the first-best case, but show that Arrow sufficiency may be violated when the control set is constrained.

The Arrow sufficiency conditions are based on the optimized Hamiltonian, which is obtained after solving the following first-order conditions

(5) $\quad \frac{\partial H}{\partial c^{i}}=H_{c i}=W_{c i}(\mathbf{s}, \mathbf{c})-\lambda^{\mathbf{T}}=0, c^{i} \in \Omega$

for the control relations $c^{x^{*}}(\mathbf{s}, \lambda)$ and $c^{y^{*}}(\mathbf{s}, \lambda)$ in the first-best case and $c^{x^{* *}}(\mathbf{s}, \lambda)$ in the second-best case. The control relations are unique given our assumption that $W$ is strictly concave in the controls.

Moreover, for any given state vector $\mathbf{s}$ and co-state vector $\lambda, c^{x^{*}}(\mathbf{s}, \boldsymbol{\lambda})=c^{x^{* *}}(\mathbf{s}, \boldsymbol{\lambda})$ since the problem is additively separable in the controls. We can further characterize the control relations by the implicit function theorem ${ }^{20}$

$$
\begin{aligned}
& \frac{\partial c^{x^{*}}}{\partial s^{x}}=\frac{\partial c^{x^{* *}}}{\partial s^{x}}=\frac{-H_{c x, s x}}{H_{c x, c x}}, \frac{\partial c^{x^{*}}}{\partial s^{y}}=\frac{\partial c^{x^{* *}}}{\partial s^{y}}=0 \\
& \frac{\partial c^{y^{*}}}{\partial s^{x}}=0, \frac{\partial c^{y^{*}}}{\partial s^{y}}=\frac{-H_{c y, s y}}{H_{c y, c y}} .
\end{aligned}
$$

The control relations can be substituted back into the Hamiltonian to derive the optimized Hamiltonian, written as $H^{*}(\mathbf{s}, \lambda)=H\left(\mathbf{s}, \mathbf{c}^{*}(\mathbf{s}, \lambda), \lambda\right)$ in the first-best case and as

\footnotetext{
${ }^{20}$ Substitute the optimized control relations back into (5) to obtain $H_{c i}(\mathbf{s}, \mathbf{c}(\mathbf{s}, \lambda), \lambda) \equiv 0$ for $c^{i} \in \Omega$. Then (6) and (7) arise from differentiating these expressions with respect to the states, after invoking our additive separability assumption (which implies $H_{c x, c y}=0$ ).
} 
$H^{* *}(\mathbf{s}, \lambda)=H\left(\mathbf{s}, c^{x^{* *}}(\mathbf{s}, \lambda), \lambda, \bar{c}^{y}\right)$ in the second-best case. Recall that the Arrow sufficiency condition guarantees a unique solution (including a unique long-run outcome) when the optimized Hamiltonian, $H^{*}(\mathbf{s}, \lambda)$ or $H^{* *}(\mathbf{s}, \lambda)$, is strictly concave in the states when $\lambda^{x}(t)=\lambda^{x^{*}}(t)$ and $\lambda^{y}(t)=\lambda^{\gamma^{*}}(t)$. Thus, the Hessian of the optimized Hamiltonian must be negative definite, requiring that the following conditions to hold along an optimal path

$$
\frac{d^{2} H^{*}}{\left(d s^{x}\right)^{2}}=H_{s x, s x}+H_{s x, c x} \frac{\partial c^{x}}{\partial s^{x}}=H_{s x, s x}+\underbrace{\frac{\left(H_{s x, c x}\right)^{2}}{-H_{c x, c x}}}_{+}<0
$$

$$
\begin{aligned}
& \frac{d^{2} H^{*}}{\left(d s^{y}\right)^{2}}=H_{s y, s y}+H_{s y, c y} \frac{\partial c^{y}}{\partial s^{y}}=H_{s y, s y}+\underbrace{\frac{\left(H_{s y, c y}\right)^{2}}{-H_{c y, c y}}}_{+}<0 \\
& \frac{d^{2} H^{*}}{\left(d s^{x}\right)^{2}} \frac{d^{2} H^{*}}{\left(d s^{y}\right)^{2}}>\left(\frac{d^{2} H^{*}}{d s^{x} d s^{y}}\right)^{2}
\end{aligned}
$$

$$
\Rightarrow \quad\left(H_{s x, s x}+\frac{\left(H_{s x, c x}\right)^{2}}{-H_{c x, c x}}\right)\left(H_{s y, s y}+\frac{\left(H_{s y, c y}\right)^{2}}{-H_{c y, c y}}\right)>\left(H_{s x, s y}\right)^{2}
$$

The sum of the terms, rather than each individual term, in conditions (8) and (9) must be negative to ensure concavity. Our assumptions about $H$ being concave in the controls implies that $-H_{c i, c i}>0$ for $i$ $\in\left\{c^{x}, c^{y}\right\}$, so that the final RHS terms in (8) and (9) are positive, as indicated. This means concavity of $H^{*}$ in the states requires $H_{s i, s i}$ for $i \in\left\{c^{x}, c^{y}\right\}$ to be sufficiently negative so as to outweigh the remaining terms in (8) and (9). As indicated above, we assume this is true in the first-best case and provide a numerical example below where this assumption holds. Condition (10) represents the determinant of the Hessian of $H^{*}$, which must be positive for the Hessian to be negative definite. Specifically, condition (10) requires the second term in the determinant, which involves cross effects, to not exceed the first term. Again, we assume this to be the case. 
We illustrate the above results using Drury's and Lodge's (2009) ecological model of intraguild predation: $G^{x}(\mathbf{s})=s^{x}\left(1-s^{x}-0.7 s^{y}\right)-\left(0.075 s^{y}\left[s^{x}\right]^{2}\right) /\left(0.01+\left[s^{x}\right]^{2}\right)$, and $G^{y}(\mathbf{s})=1.5 s^{y}\left(1-0.9 s^{x}-s^{y}\right)-$ $0.01\left(0.075 s^{y}\left[s^{x}\right]^{2}\right) /\left(0.01+\left[s^{x}\right]^{2}\right)$. This specification implies predation of each species on the other, as well as inter-species competition. The ecological relation $\boldsymbol{G}$ is not strictly convex, with the determinant of the Hessian of $G^{x}$ being negative for all $s^{x}, s^{y}$ (and, more generally, for all parameter values). Indeed, multiple equilibria exist in cases with no human intervention (i.e., with strong management banning harvest; $c^{s}=C^{y}=0$ ), as depicted in Figure 1 . There are three equilibria, $A^{0}, B^{0}$, and $C^{0}$, with $A^{0}$ and $C^{0}$ being stable nodes and $B^{0}$ being a saddle. The separatrices, denoted $Z^{0}$, represent an ecological threshold that divides the state space into two basins of attraction.

The economic model is qualitatively similar to Horan et al. (2011), with two exceptions. First, we assume that harvests of each species are valuable for consumption, whereas Horan et al. (2011) assume harvests $c^{y}$ have no consumption value. Second, we assume here that willingness to pay for consumption of harvests is nonlinear (rather than linear). ${ }^{21}$ Specifically, the total willingness to pay for consumption of harvests is $3\left(c^{x}\right)^{0.5}+1.5\left(c^{y}\right)^{0.5}$. Harvesting costs take the Schaefer specification, with total costs being $5 c^{x} / s^{x}+1.2 c^{y} / s^{y}$. Although the willingness to pay associated with species $\mathrm{x}$ is twice that of species $y$ (given a particular harvest level), the unit cost of harvesting species $x$ is more than four times larger, given identical population levels. This implies that species $y$ may have a greater net value over

\footnotetext{
${ }^{21}$ The Mangasarian conditions are never satisfied in a linear control problem because the second derivative of the control vanishes. The Arrow sufficiency conditions can only be evaluated after deriving rules for the controls that take the form $c(s, \lambda)$. The linear nature of the problem often prevents such a derivation. Rather, it is generally only possible to derive a feedback rule of the form $c(s)$. Implicitly, this rule is of the form $c(s) \equiv c(s, \lambda(s))$, but it is generally not possible to separately identify the effects of $s$ and $\lambda$. Moreover, the rule $c(s)$ is not continuously differentiable because it involves combinations of singular and non-singular solutions for each control. $A$ third complication is that the generalized Legendre-Clebsh condition, which is sufficient for nonlinear models, is only a necessary condition for linear control problems (this is discussed in Horan et al. 2011). Sufficiency is much easier to work with in the nonlinear model because the relation $c(s, \lambda)$ comes directly from the first order condition. We are confident that our intuition extends to linear control problems, but it would much more difficult [impossible] to work with sufficiency conditions.
} 
much of the state space. The resource manager is assumed to apply a discount rate of $r=0.05$.

Although $\boldsymbol{G}$ exhibits non-convexities, the economically efficient strategy yields a unique longrun, steady state equilibrium $\left(s^{x^{*}}=0.69, s^{y^{*}}=0.31, c^{x^{*}}=0.04, c^{y^{*}}=0.03, \lambda^{x^{*}}=0.34, \lambda^{y^{*}}=0.44\right)$ that satisfies the Arrow sufficiency conditions with the first principle minor $d^{2} H^{*} /\left(d s^{x}\right)^{2}=-0.74, d^{2} H^{*} /\left(d s^{y}\right)^{2}=$ -1.57 , and the determinant of the Hessian of $H^{*}$ equaling 0.46 . In particular, these results are driven by the fact that $H_{s i, s i}$ for $i \in\left\{c^{x}, c^{y}\right\}$ are sufficiently negative at the steady state, which the theory suggests must be the case for the Hamiltonian to be strictly concave in the presence of ecological nonconvexities. Comparing the unique steady state with Figure 1 indicates that optimal management endogenously alters the topology of the state space, effectively shifting the ecological threshold to the left and out of the positive orthant so that only a single basin of attraction remains (analogous to that of Horan et al. (2011)). This basin involves a relatively large amount of species $x$, since smaller levels imply larger unit harvest costs, and a relatively smaller amount of species $y$, which has been depleted somewhat due to its higher net marginal value.

In the first-best case, relaxing the Mangasarian conditions does not affect the sufficiency conditions for feasible path controllability and decouplability: these efficiency-enhancing properties still hold in the present case. Moreover, while neither path controllability nor decouplability guarantee a unique solution (i.e., a globally optimal long-run steady state outcome that can be attained regardless of the initial state $\mathbf{s}_{0}$ ), they certainly would seem to help facilitate such an outcome. Indeed, it is worth emphasizing that the Arrow sufficiency conditions ensuring a unique global optimum are satisfied despite the non-convex ecological relationship that yields multiple ecological equilibria in Fig. 1. This demonstrates the potential importance of institutional arrangements and resource allocation mechanisms for determining the existence of thresholds: efficient management can potentially eliminate thresholds from coupled systems. These results also demonstrate that the thresholds themselves are not primitives of the coupled system. 
We now turn our attention to the second-best case, which in general is more realistic since the ubiquity of ecosystem externalities implies the first-best case is unlikely - managers generally do not have enough policy tools to manage (let alone identify) each ecological state variable. In our numerical example this could be because the manager is a state official and federal government has prohibited the harvest of $s^{y}$. Indeed, a common case may be uncoordinated management by two separate government agencies. The following section 4.2 examines whether the loss of an active control can affect whether (8)-(10) are satisfied when the Mangasarian conditions do not hold.

\subsection{The second-best problem without Mangasarian sufficiency}

We now examine the concavity conditions (8)-(10) when $\Omega=\left\{c^{x}\right\}$. These conditions are rewritten for the present case below, although in contrast to our discussion in section 4.1 we do not make any a priori assumptions that the given inequalities are satisfied

$$
\frac{d^{2} H^{*}}{\left(d s^{x}\right)^{2}}=H_{s x, s x}+H_{s x, c x} \frac{\partial c^{x}}{\partial s^{x}}=H_{s x, s x}+\underbrace{\frac{\left(H_{s x, c x}\right)^{2}}{-H_{c x, c x}}}_{+}<0
$$

$$
\begin{aligned}
& \frac{d^{2} H^{*}}{\left(d s^{y}\right)^{2}}=H_{s y, s y}<0 \\
& \left(H_{s x, s x}+\frac{\left(H_{s x, c x}\right)^{2}}{-H_{c x, c x}}\right)\left(H_{s y, s y}\right)>\left(H_{s x, s y}\right)^{2}
\end{aligned}
$$

The only visible difference between conditions (11)-(13) and conditions (8)-(10) is that the partials involving $c^{y}$ are not part of the second-best conditions (11)-(13). But there may be additional effects embedded within the remaining terms, and so it is necessary to consider each condition separately.

Consider condition (11). Our assumption that $H_{s x, c y}=0$ implies that none of the remaining terms in this condition are affected by the loss of $c^{y}$ as a control. Hence, at least for any particular combination of states and controls, this condition is unchanged in the second-best case. 
Condition (12) exhibits one less positive term than its counterpart (9), suggesting that condition (12) is more likely to be satisfied in the second-best case. However, this effect may be offset by the remaining RHS term in (12), $H_{s y, s y}$, which could be significantly affected by the loss of $c^{y}$ as a control. Indeed, it is possible that $H_{s y, s y}$ could increase in value - and possibly even be positive - when $c^{y}=\bar{c}^{y}$. For example, let $\bar{c}^{y}=0$, and assume that $\left.W_{s y}\right|_{c y=0}=0$ (e.g., $s^{y}$ only affects $W$ via impacts on costs of harvesting $c^{y}$, as with our numerical example for the first-best case). Then, $\left.H_{s y, s y}\right|_{c y=0}=\lambda^{x} G_{s y, s y}^{x}+\lambda^{y} G_{s y, s y}^{y}$ could be positive for some states if $\boldsymbol{\lambda}^{\top} \mathbf{G}$ is not negative definite when $\boldsymbol{\lambda}=$ $\lambda^{* *}$. In contrast, the first-best value $\left.H_{s y, s y}\right|_{c y=c y^{*}}=W_{s y, s y}+\lambda^{x} G_{s y, s y}^{x}+\lambda^{y} G_{s y, s y}^{y}$ may be negative for all states due to the term $W_{s y, s y}<0$.

Finally, consider condition (13). The first LHS parenthetical term, which is the same as condition (11), is relatively unchanged from its counterpart in condition (10). The second LHS parenthetical term, which is the same as condition (12), is ambiguous in magnitude relative to its counterpart in condition (10). However, as argued above, it is possible that this term could be smaller or even negative in the second-best case. A smaller value would make condition (13) less likely to hold, whereas a negative value for this term would ensure that (13) does not hold.

We illustrate these results by showing that the management problem for our earlier numerical example is non-convex for the restricted control set $\Omega=\left\{c^{x}\right\}$. We assume $c^{y}=0$, such that harvesting of population $s^{y}$ is prohibited and $G^{y}$ is unaffected by direct human intervention. For example, it could be that the social planner is a state regulator, but a federal regulator closes all harvests for species $s^{y}$ due to this population being threatened or endangered. We will show this case illustrates the importance of controllability and decouplability, as the federally-imposed restrictions result in the state regulator not having sufficient control over the system.

Figure 2 depicts a feedback control diagram for the second-best example, with the optimal 
harvest $c^{x}$ embedded as a feedback function of the form as $c x^{* *}=c^{x^{* *}}\left(s^{x}, s^{y}\right)$. The null-clines, sample trajectories, and solution were computed using dynamic programming based on a value function approximation using Chebychev polynomials with a collocation approach (Fenichel et al., 2014; Miranda and Fackler, 2002); see the Appendix for details. The equilibria labeled $\tilde{A}, \widetilde{B}, \tilde{C}, \widetilde{D}$ are based on the approximation, while the actual equilibrium points, labeled $A^{* *}, B^{* *}, C^{* *}, D^{* *}$, are shown for comparison (the Appendix provides other diagnostics on the approximation precision). Note that $A^{* *}=\tilde{A}$. There are slight approximation errors associated with the other equilibria, but these are not substantial, and we note that the stability properties of each approximated equilibrium are the same as those of the corresponding actual equilibrium. For simplicity, we make reference to the approximated equilibria since the dynamics in Figure 2 are based on these.

Steady states $\tilde{A}$ and $\tilde{C}$ are stable nodes, whereas $\widetilde{B}$ and $\widetilde{D}$ are saddles (with the separatrices for $\widetilde{D}$ lying on the horizontal axis, so that $\widetilde{D}$ is effectively an unstable node when $\left.s^{y}(0)>0\right)$. The basins of attraction containing the stable nodes can be Pareto-ranked based on the economic welfare at the associated steady states. We refer to the basin containing $\tilde{C}$ as the desirable basin and the basin containing $\tilde{A}$ as the undesirable basin. This is because steady state welfare at $\tilde{C}$ is greater than steady state welfare at $\tilde{A}$, consistent with $\tilde{C}$ being closer to the first-best steady state $A^{*}$ (depicted for comparison). However, this Pareto-ranking only makes sense if society could freely choose to be in one basin or the other. In reality, the initial state $\mathbf{s}_{0}$ is given, and society may prefer to move to $\tilde{A}$ from some initial states $\mathbf{s}_{\mathbf{0}}$. In particular, the separatrices associated with $\widetilde{B}$, denoted $Z^{* *}$, represent an indifference threshold that divides the state space into two basins of attraction. Initial states that lie above and left of the threshold optimally progress to $\tilde{A}$, whereas initial states below the threshold optimally progress to $\tilde{C}$. Relative to the first-best steady state, depicted as point $A^{*}$ for comparison, population $s^{x}$ has been reduced and population $s^{y}$ increased at steady state $\tilde{C}$ (i.e., $s^{x^{* *}}<s^{x^{*}}, s^{y^{* *}}>s^{y^{*}}$ ). This result is consistent with the loss of the active control $c^{y}$ in the second-best case. 
Multiple equilibria arise in Figure 2 because the second-best problem (i) is not FPC, so that movement across the state space is restricted, and (ii) is not decouplable, so that independent control of the two states with the single control is not possible. Together, these features of the problem increase the costs of certain types of movements across the state space and enable the underlying nonconvexities associated with $\boldsymbol{G}$ to play a more prominent role: managing the bioeconomic system to override the ecological non-convexities and multiple equilibria, which is what occurred in the first-best solution, becomes prohibitively costly in the second-best case. Therefore, a globally preferred trajectory does not arise. Indeed, with no control available to manage $\mathrm{s}^{y}$, the null-cline for this population is the same as the null-cline in Figure 1. This means the ecological dynamics from Figure 1 are relevant here such that the ecological non-convexities inherent in $\mathbf{G}$ (i.e., when $c^{x}=c^{y}=0$ ) take on a more prominent

role. For instance, if the system initially sat in the neighborhood of point $A^{0}$ (as defined in Figure 1 ), then the system will remain stuck in that neighborhood. The reason is that the available management tools cannot reduce the predation on $s^{x}$ to overcome the ecologically-driven basin of attraction in this region and allow $s^{x}$ to grow; harvests $c^{x}$ only exacerbate this problem. Indeed, the basin of attraction for $A^{* *}$ in Figure 2 exceeds that of Figure 1 , due to $c^{x}$ being positive in the second-best outcome, as $Z^{* *}$ in Figure 2 lies everywhere to the right of $Z^{0}$ in Figure 1 . The non-convexities and multiple equilibria arising in the second-best problem therefore results from fewer active controls than states (a violation of the Tinbergen rule) combined with the underlying ecological dynamics being convex-concave and exhibiting multiple equilibria in the case of no human intervention (Figure 1).

\section{The potential fallacy of uniqueness}

A key result from our theoretical analysis and numerical example is that restrictions on the set of controls can generate bioeconomic multi-stability that reduces welfare in non-marginal ways. The implication is that there are potential gains from improved understanding of ecosystem linkages and/or 
institutional reforms that that lead to improved controls. The notion of institutional reforms is particularly relevant given the growing interest in polycentric governance, which involves multiple, independent centers of decision making (Ostrom, 2010a, b). Our results suggest polycentric forms of governance may only be effective at provisioning ecological services in cases where the system remains path controllable and decouplable (even after accounting for the possible ill-effects of politicaleconomic forces at play).

It would be wrong, however, to conclude that uniqueness is always preferred to multi-stability. Properties such as uniqueness or economic multi-stability (i.e., the existence of indifference thresholds that divide the state space into basins of attractions exhibiting Pareto-ranked long-run outcomes) should not be used to rank institutional arrangements.

Consider a final extension of our numerical example to illustrate a case where our second-best multi-stable outcome may be preferred to a unique outcome. Suppose that, rather than just banning harvests of population $s^{y}$ (i.e., $\bar{c}^{y}=0$ ) the federal government implements a constant stocking program for species $s^{y}$, denoted $q$, so that the population dynamics for this species become $\dot{s}^{y}=G^{y}(s)+q .{ }^{22}$ We assume $q=0.05$. Further, to facilitate comparison with the earlier results, suppose the cost of the stocking program was negligible. This scenario yields a unique basin of attraction with a smaller steady state population $s^{x}$ and a larger steady state population $s^{y}$ than occurs at steady state $\tilde{A}^{* *}$ in Figure 2 , with less economic welfare. The more restrictive control set under stocking has essentially shifted the indifference threshold from Figure 2 down and left beyond the positive orthant, resulting in a uniquely "undesirable" basin of attraction and steady state.

Alternatively, if we view Figure 3 as the base case, then making the choice set less restrictive (i.e., moving from a stocking regime to a simple harvest ban, as in Figure 2) shifts the "invisible" threshold in Figure 3 up and right into the positive orthant and creating a Pareto-preferred basin of

\footnotetext{
${ }^{22}$ This stocking program could also be viewed as exogenous immigration of the species.
} 
attraction. That is, the less restrictive control set creates opportunities for moving into a better basin of attraction. Making the choice set even less restrictive further shifts the threshold up and left and out of the positive orthant so that a uniquely good basin arises, as in the first-best case. Horan et al. (2011) found a similar type of result in a linear control model. The finding that welfare is improved by reducing the restrictions on the control set is consistent with LeChatelier's Principle. ${ }^{23}$

The unique basin of attraction arises in Figure 3 because, relative to Figure 2, the more restricted control set further reduces the controllability of the system - to the point that it becomes impossible to escape the undesirable basin of attraction. Welfare is subsequently reduced because the reduced ability to control the system increases the costs of control.

\section{Discussion}

Thresholds and tipping points have become major touchstones of the science-economic-policy interface (see other articles in this issue). Research in this area largely focuses on recognizing, detecting, and either adapting to or avoiding thresholds. In particular, prior economic work has stressed how ecological non-convexities can create economic thresholds that separate optimal management regimes (e.g., prevention versus adaptation). Ecological non-convexities do not always generate economic thresholds, but when they do then the choice of which long-run outcome to pursue depends on the current state of the system. This means history plays a key role in determining the best course of action, with prompt action generally preferred to delay.

Our analysis highlights how the choice set over which managers can act, as conditioned by governing institutions, can combine with ecological non-convexities to generate economic thresholds. Institutions become increasingly important once we embrace the notion that ecosystems are complex

\footnotetext{
${ }^{23}$ Fenichel (2013) points out that the less restricted control set must be able to replicate the more restricted control set for the rank ordering of controls sets to hold. For example, if responses are homogenized to "increase" control, then the seemingly more complete control set may not be able to replicate the seemingly partial control set and the control sets cannot be truly rank ordered.
} 
and involve a larger number of interactions or linkages that generate ecosystem externalities in the absence of appropriate policy tools. Efficient management generally requires using policy tools to manage each linkage, but in practice this requires institutional arrangements and capabilities that may not currently exist. This means managing systems with potential thresholds involves qualitatively enhancing the capacity of managers to act, and not simply when or how much to act within the confines of current institutions. Less efficient institutional regimes that restrict environmental managers' ability to control ecosystem externalities can either generate or adversely shift economic thresholds so that poor economic and ecological outcomes are more likely to occur. In contrast, more efficient institutional regimes that provide greater control over the ecological system also provide greater opportunities for society to avoid thresholds leading to bad outcomes. In other words, economic thresholds are institutionally-dependent and depend on managers' abilities to exhibit control over interacting states that generate ecosystem externalities. Environmental managers may therefore do better to focus on structuring institutions that enable society to pursue trajectories leading to the best possible outcomes, rather than managing based on thresholds arising under given institutional structures. Of course, as Aoki and Canzoneri (1979) point out, it is important to determine if policies directed at different features need to be coordinated, e.g., when different agencies manage different parts of the system. This means that an important part of managing thresholds is coordinating policy and examining when institutional design is overly restrictive or overly diffuse (e.g., overly polycentric). When institutional reform is not possible, then management is relegated to the second-best.

An important criterion for efficient resource management is that the number of instruments equal the number of ecosystem externalities and other market failures. This is the so-called Tinbergen rule that has guided macroeconomic policy and the management of static environmental externalities, but has not yet been applied to dynamic resource problems. Resource managers may need to be creative when developing new policy levers and instruments to satisfy the Tinbergen rule, but first they 
need to understand the nature of the externalities.

Ecological scientists have an important role to play in describing the ecological system and helping to identify targetable ecological interactions to manage. Too often economists have oversimplified the ecology to provide more tractable dynamic models (e.g., longing to prove uniqueness of solutions) without a clear understanding of how more ecological details can lead to improved management. This is true even in cases where thresholds do not arise. For example, Fenichel and Horan (2007b) find it is optimal for a disease to remain endemic within a simple wildlife disease system based on total animal abundance, but subsequently find that eradication becomes optimal if managers take advantage of increased ecological detail and target management by wildlife sex (Fenichel and Horan, 2007a). Tschirhart (2012) shows how biological interactions create non-convexity, but as ecological details are added these non-convexities may vanish when management embraces these details. Adding ecological structure in some cases may enable controls to better target ecological states, thus overcoming the non-convexity and leading to unique long-run equilibria.

It is not enough to have sufficiently descriptive ecological models; understanding the proper use of these models is also essential. Many ecological scientists and economists alike oversimplify the notion of ecological thresholds and tipping points and their role for management. Management based on "thresholds" that are computed without regard to economic behavior will generally be inefficient, and perhaps ineffective, which means such thresholds are not the relevant concepts for guiding management. Rather, management strategies are optimally guided by ecological and economic interactions, with the resulting coupled system dynamics and related thresholds arising from these strategies and their underlying institutions. This means tipping behavior arises in response to management, not the other way around. Ascribing tipping dynamics solely to the ecological components of the system undermines our ability to learn about and manage ecological systems.

These results highlight the importance of how ecological-economic systems are framed. At the 
end of the day, we are working with models that simplify reality. The simplifications we choose or opportunities we assume away when developing those models, as well as the manner in which the models are used and interpreted, can influence policy advice in non-marginal ways.

\section{Appendix}

Multiple methods have been suggested for solving optimal control problems with incomplete control sets (e.g., Horan et al., 2011; Salau and Fenichel, 2015), but these approaches are restricted to problems linear in the control variables. Instead of approaching the problem in an optimal control framework, we use a continuous time dynamic programming approach that exploits the Hamilton-Bellman-Jacobi identity inspired by Balikcioglu et al. (2011), Marten and Moore (2011), and Fenichel et al. (2014). This approach enables us to use numerical value function approximation and collocation techniques to recover a feedback rule for the optimal control program (Miranda and Fackler, 2002). Importantly, this enables us to map trajectories as well as equilibria.

First, recognize that

(A1) $\quad r V(\boldsymbol{s})=H(\boldsymbol{s}, \boldsymbol{c}, \boldsymbol{\lambda})=W(\boldsymbol{s}, \boldsymbol{c})+\lambda^{T} \boldsymbol{G}(\boldsymbol{s}, \boldsymbol{c})$

and that $\lambda=\nabla_{s} V$.

Second, we approximate $V(\boldsymbol{s}) \approx \mu(\boldsymbol{s})=\sum_{n=0}^{N-1} \beta_{n} \phi_{n}(\boldsymbol{s})$, where $\mu(\boldsymbol{s})$ is a two-dimensional Chebyshev polynomial with $N$ basis functions (Miranda and Fackler, 2002) that span the state space. The $\beta_{n}=\boldsymbol{\beta}$ coefficients determine the weighting of the $\phi_{n}$ basis functions, which are chosen to maximize the quality of the approximation. The two-dimensional Chebyshev polynomial is the tensor product of one-dimensional Chebyshev polynomials. The combination of Chebyshev nodes and polynomials distributes the error between the approximating and unknown true function evenly, resulting in the best 
polynomial specification for functional approximation (Press et al., 2007). We find the $5^{\text {th }}$ order polynomials, making $N=25$, works sufficiently well (see diagnostics below).

Third, we recognize the optimal solution must satisfy Eq (5) from the main text. Imposing this condition enables us to write

(A2) $\quad c^{i^{*}}=f(s, \lambda)$

In the fourth step, we make use of Fenichel's and Abbotts's (2014b) observation the derivatives of Chebyshev polynomials are good approximations for the derivatives of the functions that the polynomials are being used to approximate and making appropriate substitutions into (A1) state that a perfect approximation would lead to

$$
\Phi=r \mu(\boldsymbol{s})-\left[W\left(\boldsymbol{s}, f\left(\boldsymbol{s}, \nabla_{\boldsymbol{s}} \mu(\boldsymbol{s})\right)\right)+\nabla_{\boldsymbol{s}} \mu(\boldsymbol{s})^{T} \boldsymbol{G}\left(\boldsymbol{s}, f\left(\boldsymbol{s}, \nabla_{\boldsymbol{s}} \mu(\boldsymbol{s})\right)\right]=0\right.
$$

The function $\Phi$ is only a function of state variables and unknown Chebyshev coefficients, $\boldsymbol{\beta}$. We construct a grid of collocation nodes, with the nodes distributed as the roots of a Chebyshev polynomial over the state space. In our numerical application we use a grid based on a $30^{\text {th }}$ order polynomial yielding 900 nodes. Each node as unique value of $\Phi$. We choose $\boldsymbol{\beta}$ to minimize $\Phi^{T} \Phi$, such that $\Phi \approx 0$. The Appendix Figures show the distribution of residual error (Figure A1) and where those errors are located in relation to the feedback control diagrams in the main text (Figure A2). Furthermore, the quality of the approximation can be inferred from the accuracy of the equilibria. All equilibria have the same stability characteristics as the analytically derived equilibria.

\section{References}

Aoki, M., 1975. On a Generalization of Tinbergen's Condition in the Theory of Policy to Dynamic Models. The Review of Economic Studies, 42, 293-296.

Aoki, M., Canzoneri, M., 1979. Sufficient Conditions for Control of Target Variables and Assignment of Instruments in Dynamic Macroeconomic Models. International Economic Review, 20, 605-616. Balikcioglu, M., Fackler, P.L., Pindyck, R.S., 2011. Solving optimal timing problems in environmental economics. Resource and Energy Economics, 33(3), 761-768. 
Beisner, B.E., Haydon, D.T., Cuddington, K., 2003. Alternative stable states in ecology. Frontiers in Ecology and the Environment, 1(7), 376-382.

Biggs, R., Carpenter, S.R., Brock, W.A., 2009. Turning back from the brink: detecting an impending regime shift in time to avert it. Proceedings of the National Academy of Sciences, 106(3), 826831.

Brock, W., Xepapadeas, A., 2002. Optimal ecosystem management when species compete for limiting resources. Journal of Environmental Economics and Management, 44, 189-220.

Brock, W.A., Starrett, D., 2003. Managing systems with non-convex positive feedback. Environmental and Resource Economics, 26, 575-602.

Bryson, A.E., Ho, Y.C., 1975, Applied Optimal Control: Optimization, Estimation, and Control. Hemisphere Publishing, New York.

Caputo, M.R., 2005, Foundations of Dynamic Economic Analysis: optimal control theory and applications. Cambridge University Press, New York.

Caulkins, J.P., Feichtinger, G., Grass, D., Tragler, G., 2009. Optimal control of terrorism and global reputation: A case study with novel threshold behavior. Operations Research Letters, 27(6), 387391.

Chen, P., 2002. Microfoundations of macroeconomic fluctuations and the laws of probability theory: the principle of large numbers versus rational expectations arbitrage. Journal of Economic Behavior \& Organization, 49, 327-344.

Crocker, T.D., Tschirhart, J., 1992. Ecosystems, externalities, and economies. Environmental and Resource Economics, 2, 551-567.

Dasgupta, P., Maler, K.G., 2003. The economics of non-convex ecosystems: Introduction. Environmental and Resource Economics, 26, 499-525.

Davidson, R., Harris, R., 1981. Non-convexities in continuous-time investment theory. Review of Economic Studies, 48(2), 235-253.

Drury, K.L.S., Lodge, D.M., 2009. Using mean first passage times to quantify equilibrium resilience in perturbed intraguild predation systems. Theoretical Ecology, 2, 41-51.

Fenichel, E.P., 2013. Economic considerations for social distancing and behavioral based policies during an epidemic. Journal of Health Economics, 32, 440-451.

Fenichel, E.P., Abbott, J.K., 2014a. Heterogeneity and the fragility of the first best: Putting the "micro" in bioeconomic models of recreational resources. Resource and Energy Economics, 36, 351-369.

Fenichel, E.P., Abbott, J.K., 2014b. Natural capital from metaphor to measurement. Journal of the Association of Environmental and Resource Economists, 1(1), 1-27.

Fenichel, E.P., Gopalakrishnan, S., Bayasgalan, O., 2015, Bioeconomics: nature as capital. In: R. Halvorsen, D.F. Layton (Eds.). Handbook on the Economics of Natural Resources. Edward Elgar, pp. 165-205.

Fenichel, E.P., Horan, R.D., 2007a. Gender-based harvesting in wildlife disease management. American Journal of Agricultural Economics, 89(4), 904-920.

Fenichel, E.P., Horan, R.D., 2007b. Jointly-determined ecological thresholds and economic trade-offs in wildlife disease management. Natural Resource Modeling, 20(4), 511-547.

Fenichel, E.P., Horan, R.D., Bence, J.R., 2010. Indirect management of invasive species with bio-control: a bioeconomic model of salmon and alewife in Lake Michigan. Resource and Energy Economics, 32, 500-518.

Fenichel, E.P., Richards, T.J., Shanafelt, D., 2014. The control of invasive species on private property with neighbor-to-neighbor spillovers. Environmental and Resource Economics, 59(2), 231-255.

Gotelli, N.J., 2008, A Primer of Ecology. Sinauer Associates, Sunderland, MA.

Groffman, P.M., Baron, J.S., Blett, T., Gold, A.J., Goodman, I., Gunderson, L.H., Levinson, B.M., Palmer, M.A., Paerl, H.W., Peterson, G.D., Poff, N.L., Rejeski, D.W., Reynolds, J.F., Turner, M.G., 
Weathers, K.C., Wiens, J., 2006. Ecological thresholds: the key to successful environmental management or an important concept with no practical application? Ecosystems, 9, 1-13.

Horan, R.D., Bulte, E.H., 2004. Optimal and open access harvesting of multi-use species in a second-best world. Environmental and Resource Economics, 28, 251-272.

Horan, R.D., Fenichel, E.P., Drury, K.L.S., Lodge, D.M., 2011. Managing ecological thresholds in coupled environmental-human systems. Proceedings of the National Academy of Sciences, 108(18), 7333-7338.

Lenton, T.M., Held, H., Kriegler, E., Hall, J.W., Lucht, W., Rahmstorf, S., Schellnhuber, H.J., 2008. Tipping elements in the earth's climate system. Proceedings of the National Academy of Sciences, 105(6), 1786-1793.

Levin, S., Xepapadeas, A., Crepin, A.-S., Norberg, J., De Zeeuw, A., Folke, C., Hughes, T., Arrow, K., Barrett, S., Daily, G., Ehrlich, P.R., Kautsky, N., Maler, K.-G., Polasky, S., Troell, M., Vincent, J.R., Walker, B., 2013. Social-ecological systems as complex adaptive systems: modeling and policy implications. Environmental and Development Economics, 18, 111-132.

Lipsey, R.G., Lancaster, K., 1956. The general theory of second best. Review of Economic Studies, 24, 1132.

Marten, A.L., Moore, C.C., 2011. An options based bioeconomic model for biological and chemical control of invasive species. Ecological Economics, 70, 2050-2061.

Melstrom, R.T., Horan, R.D., 2013. Managing excessive predation in a predator-endangered prey setting. Ecological Economics, 90, 85-93.

Miranda, M.J., Fackler, P.L., 2002, Applied Computational Economics and Finance. MIT Press, Cambridge

Mumby, P.J., Hastings, A., Edwards, H.J., 2007. Thresholds and the resilience of Caribbean coral reefs. Nature, 450(1), 98-101.

Nyberg, L., Viotti, S., 1978. Controllability and the theory of economic policy a critical view. Journal of Public Economics, 9, 73-81.

Ostrom, E., 2010a. Beyond markets and states: Polycentric governance of complex economic systems. American Economic Review, 100, 641-672.

Ostrom, E., 2010b. Polycentric systems for coping with collective action and global environmental change. Global Environmental Change, 20(4), 550-557.

Press, W.H., Teukolsky, S.A., Vetterling, W.T., Flannery, B.P., 2007, Numerical Recipes 3rd Edition: The Art of Scientific Computing. Cambridge University Press, New York.

Rondeau, D., 2001. Along the way back from the brink. Journal of Environmental Economics and Management, 42, 156-182.

Salau, K.R., Fenichel, E.P., 2015. Bioeconomic analysis supports the endangered species act. Journal of Mathematical Biology, 71, 817-846.

Scheffer, M., Carpenter, S.R., 2003. Catastrophic regime shifts in ecosystems: linking theory to observation. TRENDS in Ecology and Evolution, 18(12), 648-656.

Skiba, A.K., 1978. Optimal growth with convex-concave production function. Econometrica, 46(3), 527539.

Starrett, D.A., 1972. Fundamental noncovexities in the theory of externalities. Journal of Economic Theory, 4, 180-199.

Tahvonen, O., Salo, S., 1996. Nonconvexities in optimal pollution accumulation. Journal of Environmental Economics and Management, 31, 160-177.

Tinbergen, J., 1956, Economic policy: principles and design. North-Holland Publishing Company, Amsterdam.

Tschirhart, J., 2012. Biology as a source of non-convexities in ecological production functions. Environmental and Resource Economics, 51, 189-213. 
Wilen, J.E., 1985, Bioeconomics of renewable resource use. In: A.V. Kneese, J.L. Sweeney (Eds.). Handbook of Natural Resource and Energy Economics. North-Holland, New York, pp. 61-124.

Wirl, F., 1992. Cyclical strategies in two-dimensional optimal control models: necessary conditions and existence. Annals of Operations Research, 37, 345-356.

Wirl, F., 2004. Thresholds in concave renewable resource models. Ecological Economics, 48, 259-267.

Wirl, F., Feichtinger, G., 2005. History dependence in concave economies. Journal of Economic Behavior \& Organization, 57, 390-407. 
Table 1. Non-convexities in ecological systems

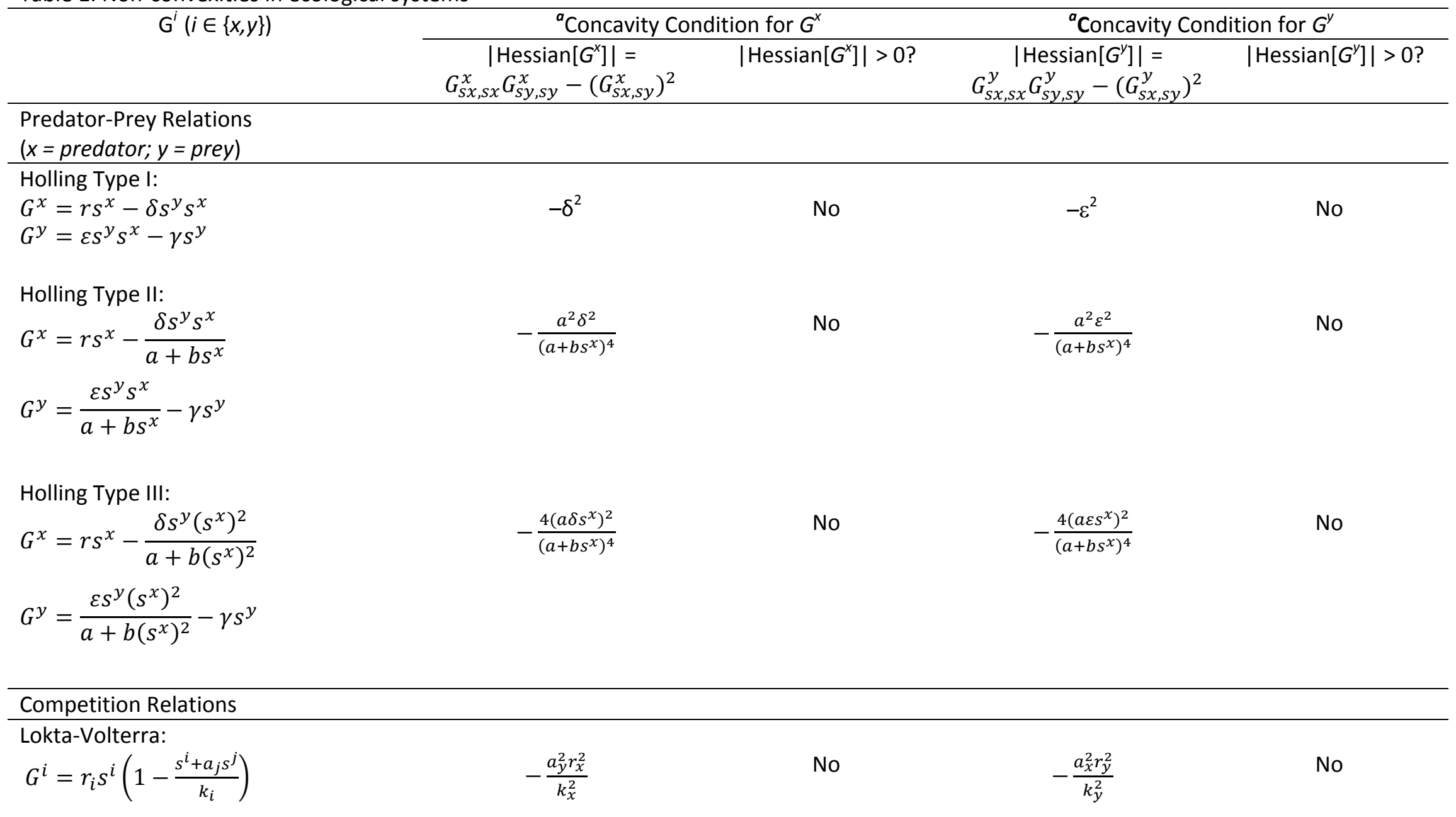

${ }^{a} G^{i}$ (for $i \in\{x, y\}$ ) should be concave for valuable resource species, $\lambda^{i}>0$, and convex for nuisance species to promote concavity of the Hamiltonian. The determinant condition in the table is for concavity, whereas the opposite must hold for convexity. Note that the determinant conditions can only support convexity. However, an additional requirement for convexity is a positive first principle minor, i.e., $G_{x x}{ }^{i}>0$, but we find this relation is negative in each case except for the type III functional response, where the sign if negative. Hence, each of the specifications is generally convex-concave. 
Figure captions

Figure 1. Phase dynamics of the ecological model.

Figure 2. Feedback control diagram showing the system dynamics conditional on the optimal program conditional on the incomplete control set (second-best).

Figure 3. Feedback control diagram showing the system dynamics conditional on the optimal program conditional on the incomplete control set (second-best) and stocking species $s^{y}$.

Figure A1 Histogram of approximation residuals.

Figure A2 Contour map of approximation residuals with null-clines for reference. 


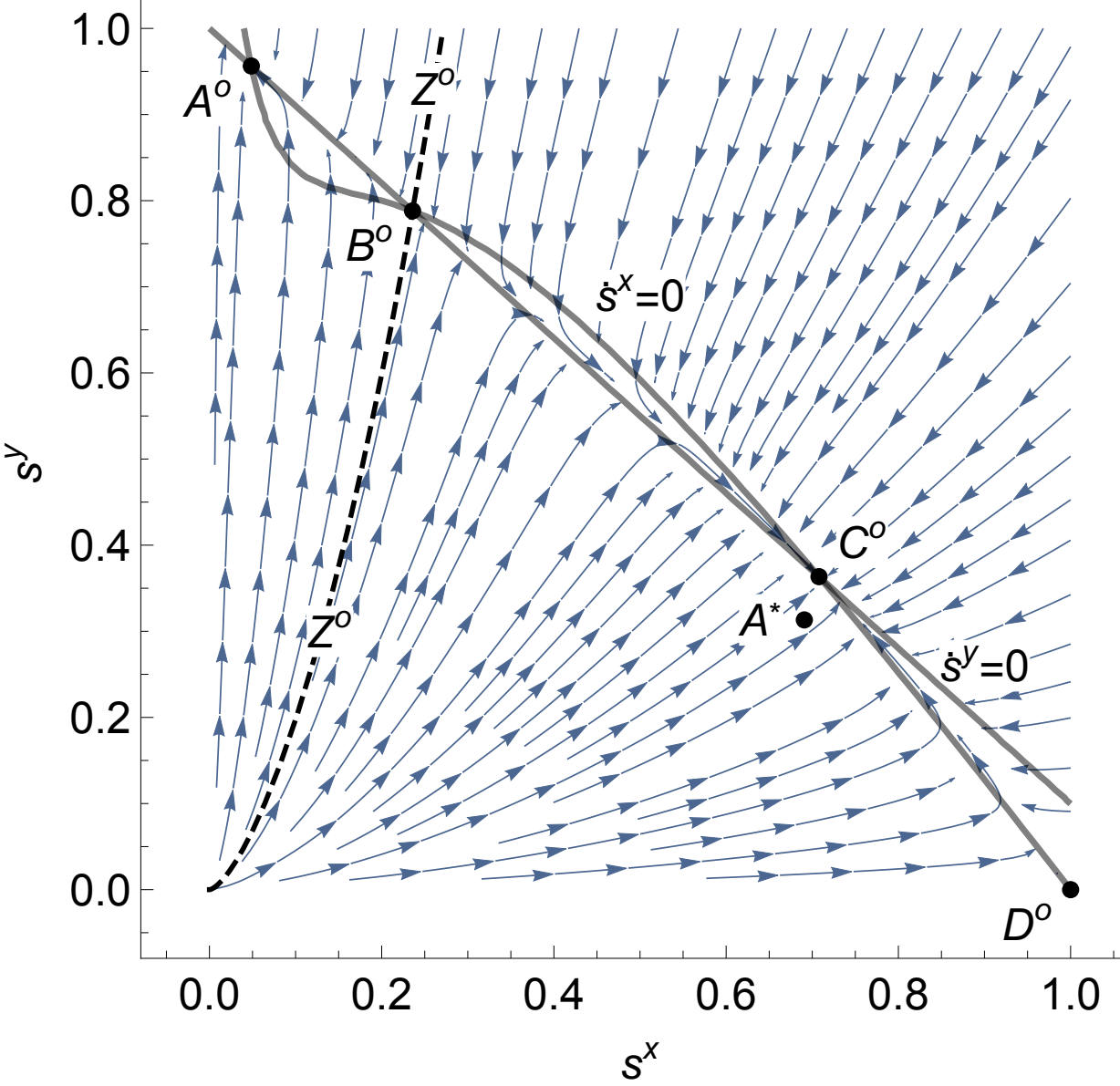




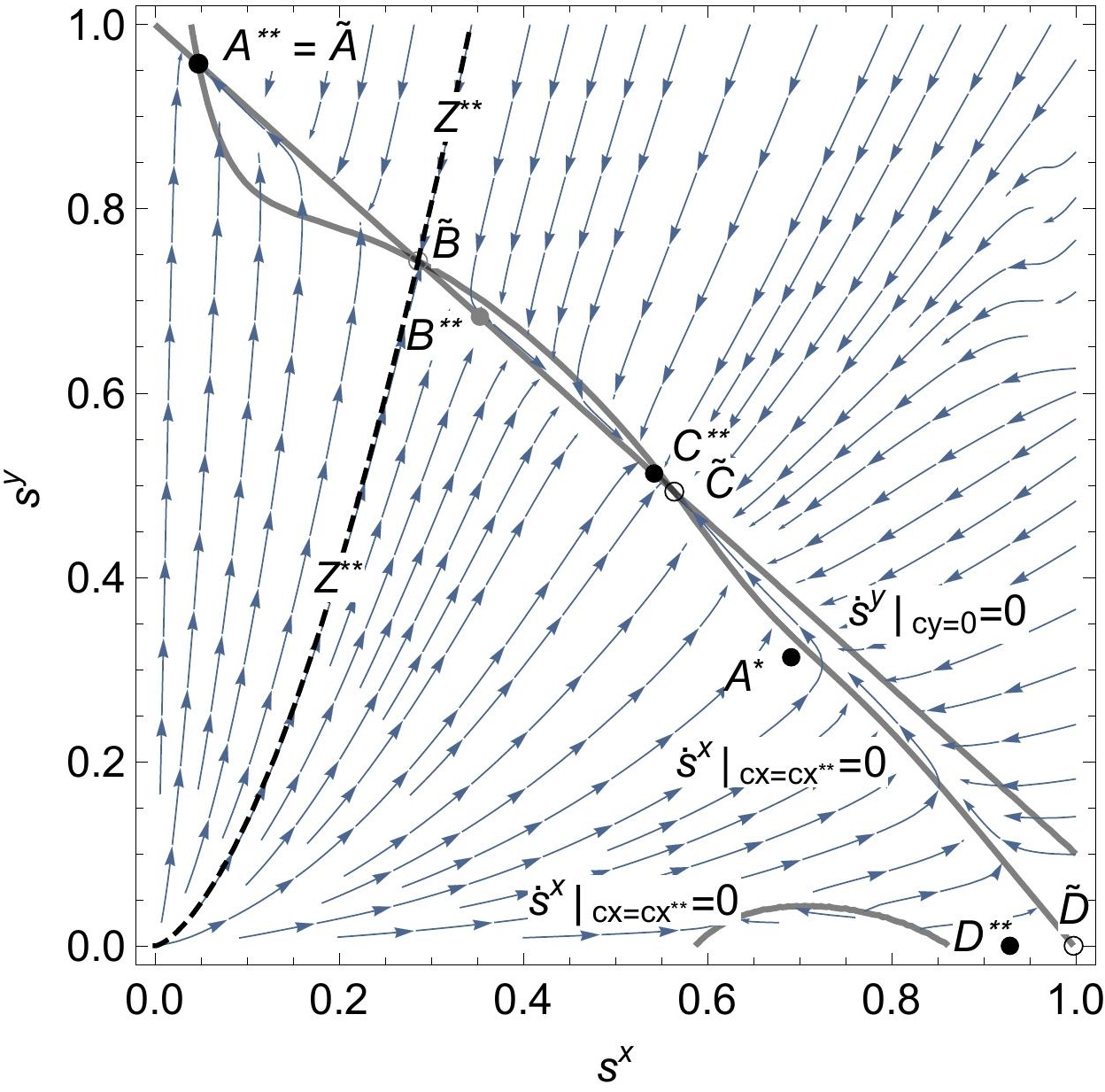




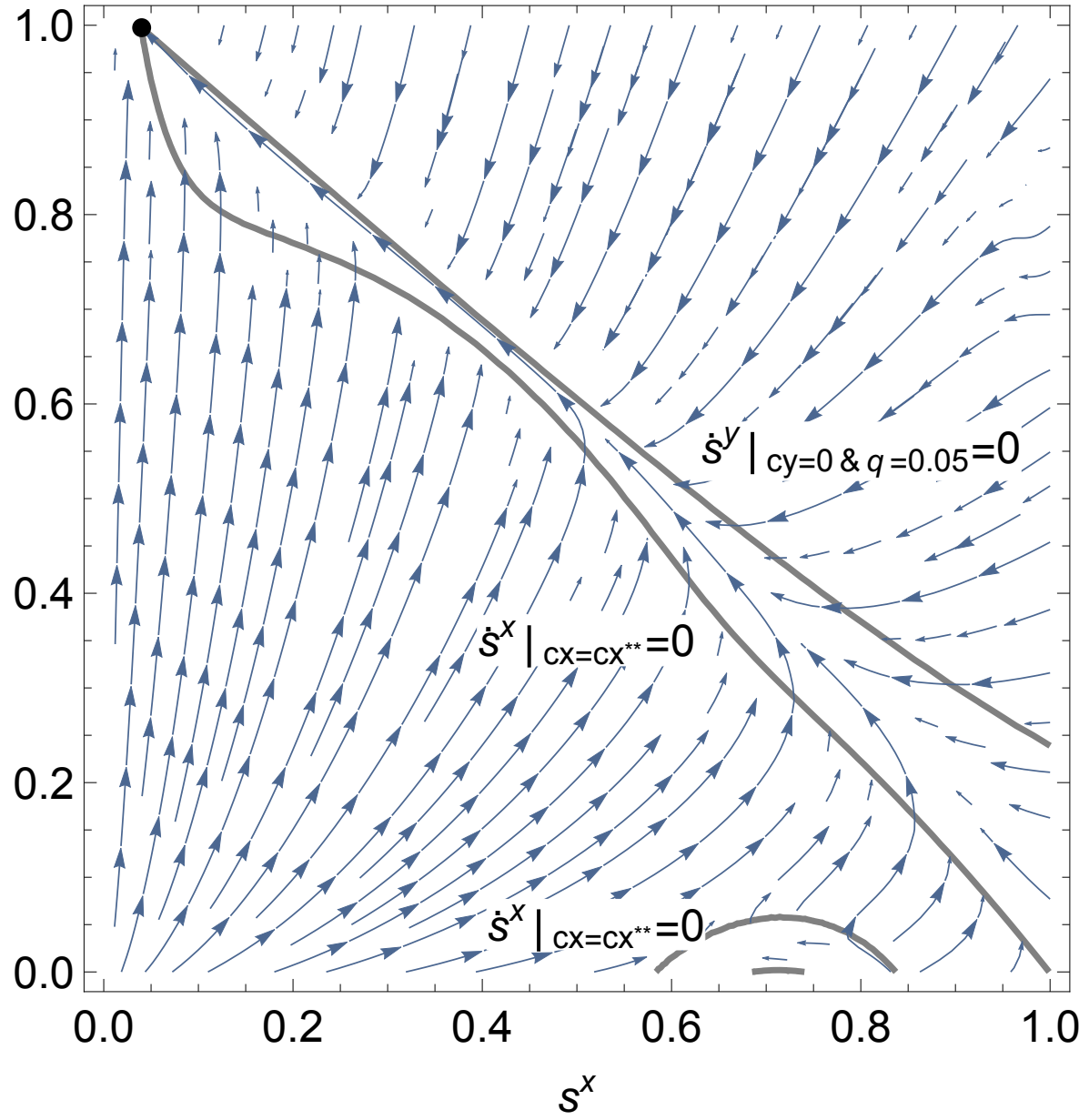




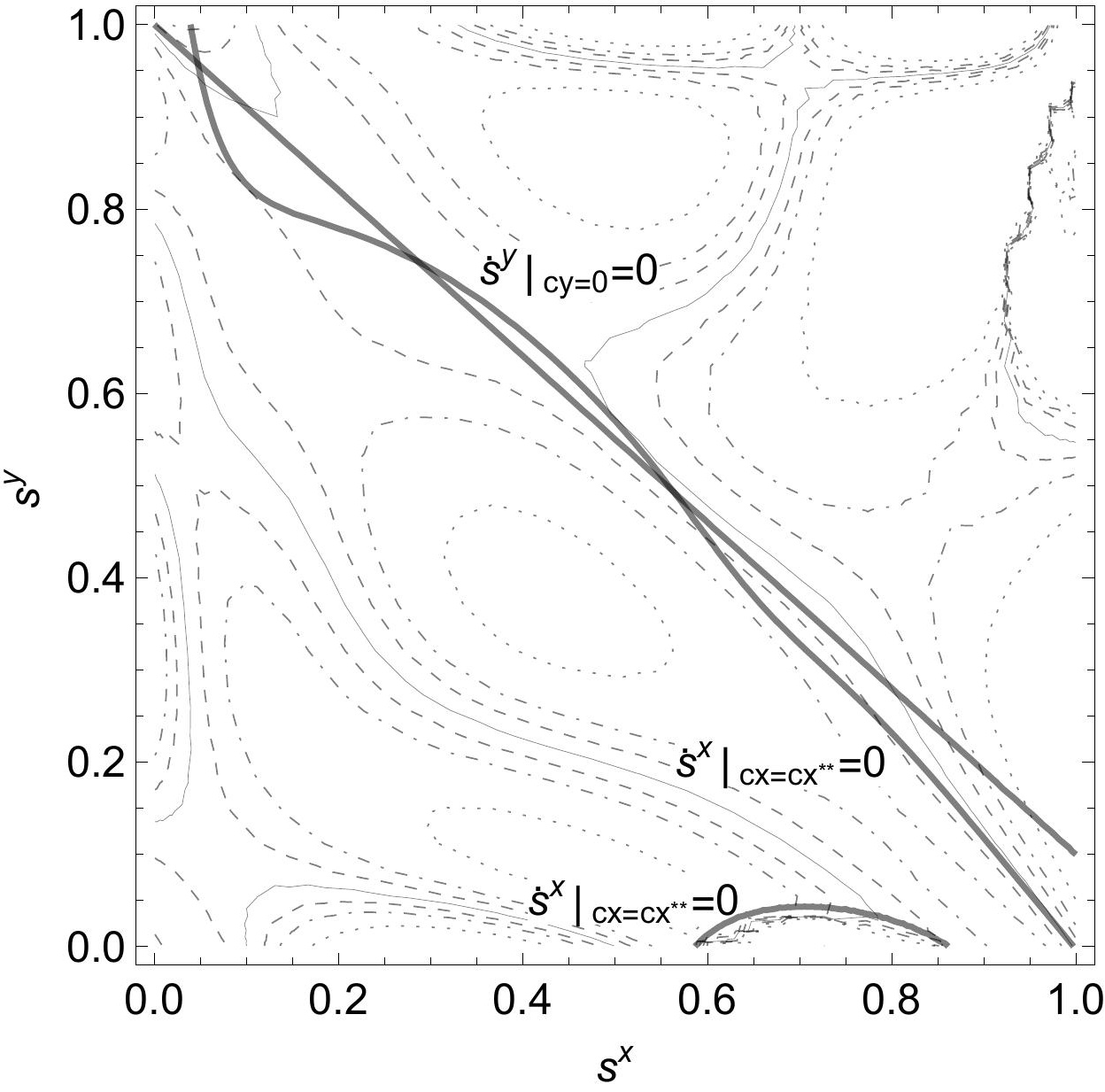

